\title{
Ueber Urethan.
}

Von Dr. G. Vulpius.

Nahezu alle in letzten Jahren, sei es versuchsweise, sei es definitir in den Arzneischatz aufgenommenen neuen Mittel waren Antiseptica, Antipyretica, Anästhetica oder endlich Hypnotica. Zu dieser letzteren Gruppe gehört auch das gegenwärtig noch im Versuchsstadium befindliche, aber, wie es scheint, zu einer nicht unwichtigen Rolle berufene Urethan oder, wie man, da Urethan eigentlich ein Gattungsname ist, genaner präcisirend sagen müsste, das Aethylurethan.

Man bezeichnet mit dem Namen der Urethane sämmtliche Aether der Carbaminsäure. Letztere ist aufzufassen als ein Amidderivat der zweibasischen Kohlensäure, worin ein Hydroxyl durch Amid crsetzt ist. Die Beziehungen der Carbaminsäure zur Kohlensäure einerseits, zum Carbamid oder Harnstoff andererseits ergeben sich aus folgender Darstellung:
$\mathrm{CO}<\mathrm{OH}$
$\mathrm{CO}<\mathrm{NH}_{\mathrm{OH}}^{2}$
$\mathrm{CO}<\mathrm{NH}^{2}$
$=$ Kohlensäure. = Carbaminsäure.
$=$ Harnstoff.

Das Ammoniumsalz der im freien Zustande noch nicht erhaltenen Carbaminsäure findet sich bekanntlich in dem käuflichen kohlensiuren Ammonium, wolches als eine Verbindung gloicher Moleküle sauren kohlensauren und carbaminsauren Ammoniums zu betrachten und dementsprechend $\mathrm{NH}^{4} \cdot \mathrm{H} \cdot \mathrm{CO}^{3}+\mathrm{NH}^{4} \mathrm{O} \cdot \mathrm{NH}^{2} \cdot \mathrm{CO} \mathrm{zu}$ schreiben ist. Man weiss ja auch längst, dass die in schlecht verschlossenen Gefässen unter Bildung eines weissen pulverigen Ueberzuges der einzelnen Stücke stattfindende Zersetzung des käuflichen kohlensauren Ammoniums auf einer Spaltung desselben in jene beiden Salze beruht, wobei das carbaminsaure Ammonium in Ammoniak und Kohlensäureanhydrid zerfällt nach der Gleichung: $\mathrm{NH}^{4} \mathrm{O} \cdot \mathrm{NH}^{2} \cdot \mathrm{CO}=2 \mathrm{NH}^{3}+\mathrm{CO}^{2}$, während saures kohlensaures Ammonium zurüchbleibt. Rascher und ohne weitere Zersetzung wird das carbaminsaure Ammonium von letzterem Begleiter getrennt durch Behandeln mit starkem. Weingeist, welcher nur jenes aufnimmt. In dor Regel wird jedoch das carbaminsaure Ammonium durch gleichzeitiges Einleiten von Koblensäureanhydrid und Ammoniak in absoluten Alkohol erhalten, wobei Wasser sorgfältig fernzuhalten ist, da sich mit ihm das Salz sofort in kohlensaures Ammon umsetzt. 
Dieses Alles übrigens nur nebenbei, denn es bildet das carbaminsaure Ammonium keineswegs den Ausgangspunkt zur Herstellung der Urethane. Man erhält letztere vielmehr auf verschiedenen anderen Wegen, wobei stets der Wasserstoff im Hydroxyl der Carbaminsäure durch ein einwerthiges Alkoholradical ersetzt erscheint. So kann man beispielsweise das Methylurethan erhalten durch Einleiten von Chlorcyan in Methylalkohol, während eine allgemeinerer Anwendung fähige Methode sich der Einwirkung von Ammoniak auf die Kohlensäureäther oder Chlorkohlensäureäther der Alkoholradicale bedient. Auf diese Weise resultirt aus Kohlensäuremethyläther und Ammoniak Methylurethan und Methylalkohol:

$$
\mathrm{CO}<\mathrm{OCH}^{3}+\mathrm{NH}^{3}=\mathrm{CO}<\frac{\mathrm{NH}^{2}}{\mathrm{OCH}^{3}}+\mathrm{CH}^{3} \cdot \mathrm{OH}
$$

und aus Chlorkohlensäuremethyläther und Ammoniak Aethylurethan und. Salzsäure:

$$
\mathrm{CO}<\underset{\mathrm{OC}^{2} \mathrm{H}^{5}}{\mathrm{Cl}}+\mathrm{NH}^{3}=\mathrm{CO}<\frac{\mathrm{NC}^{2}}{\mathrm{OC}^{5}}+\mathrm{HCl} .
$$

Der Weg, welchen die chemische Industrie einschlägt, um sich Urethan in grösseren Mengen zu verschaffen, ist jedoch keiner der bisher angegebenen, sondern es besteht derselbe in einem der Fabrikation angepassten Ausbau der Methode, welche Bunte schon 1869 in den "Annalen der Chemie und Pharmacie" angegeben hat. Nach ihm lässt man salpetersauren Harnstoff im geschlossenen Rohr bei $120^{\circ}-130^{\circ}$ einige Stunden lang auf einen mässigen Ueberschuss von Aethylalkohol einwirken. Dabei vollzieht sich folgende Reaction:

$$
\begin{aligned}
& \left.\mathrm{CON}^{2} \mathrm{H}^{4} \cdot \mathrm{NO}^{3} \mathrm{H}+\underset{\mathrm{H}}{\mathrm{C}^{2} \mathrm{H}^{5}}\right\} \mathrm{O}=\mathrm{NH}^{4} \mathrm{NO}^{3}+\mathrm{CO}<\mathrm{OC}^{2} \mathrm{HH}^{5}
\end{aligned}
$$

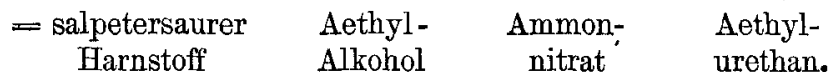

Nach dem Erkalten findet sich eine krystallinische Masse vor, welche man in Wasser löst, um dann dic Lösung mit Aether auszuschütteln. Die beim Verdunstenlassen erhaltonen Krystalle sind Urethan, welches noch durch Destillation und Umkrystalliren gereinigt wird.

Das Aethylurethan, $\mathrm{NH}^{2} . \mathrm{CO}^{2} . \mathrm{C}^{2} \mathrm{H}^{5}$, welches zur Zeit schlechtweg als Urethan bezeichnet wird, stellt ausgebildete, säulen- und tafelförmige, farblose Krystalle dar, welche beimahe geruchlos sind und einen eigenthümlichen, salpeterartigen, jedoch in Lösung weder' 
unangenehmen, noch besonders starken Geschmack besitzen. Auf $49-50^{\circ}$ erwärmt, schmelzen dieselben, es zeigt übrigens das Urethan die Erscheinung der Ueberschmelzung in ziemlich ausgeprägter Weise, so dass es, einmal geschmolzen, weit unter den angegebenen Schmelzpunkt abgekühlt werden kann, um dann bei Erschütterung u. dergl. plötzlich zu einer festen Masse zu erstarren, was bei der für die Praxis unerlässlichen Schmelzpunktsbestimmung wohl zu beachten ist. Der Siedepunkt liegt bei etwa $170^{\circ}$ und es entwickeln sich dabei Dämpfe, welche angezündet mit bläulicher Flamme brennen und sich an kalten Gegenständen zu feder- und sternförmig gruppirten Krystallen verdichten. Als vollständig und unzersetzt flüchtig ist somit das Aethylurethan destillirbar und sublimirbar. Von den gewöhnlichen Lösungsmitteln wird es leicht aufgenommen; es genügen zur Lösung von 1 Theil Urethan bei gewöhnlicher Temperatur in abgerundeten Zahlen: 1 Thl. Wasser, 0,6 Thle. Weingeist, 1 Thl. Aether, 1,3 Thle. Chloroform, 0,8 Thle. verflüssigte Carbolsäure, 3 Thle. Glycerin, 15 Thle. Ricinusöl und 20 Thle. Olivenöl. Die drei letztgenannten Lösungen sind durch Erwärmen herzustellen, scheiden aber während des Erkaltens kein Urethan aus.

In der Menge eines Tropfens auf dem Objectträger der freiwilligen Verdunstung überlassen, hinterlässt die wässerige Lösung treppenförmig aufgebaute Tafeln, die weingeistige lange, dicht aneinandergeschlossene Spiesse mit zwischengelagerten, vereinzelten, kurzen schiefen Säulen, die ätherische feingestreifte Tafeln, diejenige in Chloroform endlich zugespitzte Säulengruppen. Die wässerige Lösung des Urethans reagirt nentral gegen Lackmus. Von concentrirter Essigsäure wird Urethan in reichlicher Menge aufgenommen, ebenso von mässig verdünnten Mineralsäuren und zwar ohne bemerkenswerthe Farbenerscheinung. Die wässerige Lösung verhält sich indifferent gegen Metallsalze.

Als eigentliche Identitätsreaktion des Urethans aber kann zunächst sein Verhalten gegen concentrirte Schwefelsäure beim Erhitzen betrachtet werden. Es findet nämlich hierbei, nachdem erst Lösung eingetreten ist, heftiges Aufschäumen in Folge reichlicher und nachhaltiger Gasentwickelung statt. Das entweichende Gas röthet feuchtes Lackmuspapier und trübt Kalkwasser, ist also Kohlensäureanhydrid. Ferner entwickelt das Urethan beim Erhitzen mit Kalilauge durch den Geruch zu erkennendes und in bekannter Weise mit rothem Lackmuspapier oder Salzsäure nachweisbares Ammoniak. 
Die Prüfung des Urethans ergiebt sich aus den mitgetheilten physikalischen und chemischen Eigenschaften von selbst. Es muss in wässeriger Lösung neutral reagiren, in Wasser, sowie in Aether vollkommen löslich und beim Erhitzen ohne Rückstand flüchtig sein. Hauptsächlich jedoch wird man sein Augenmerk auf den Schmelzpunkt zu richten haben. Derselbe soll zwischen 49 und $50^{\circ}$ liegen, da man nur hierin ein einfaches Mittel besitzt, sich einerseits vor einem durch starken Feuchtigkeitsgehalt minderwerthigen Aethylurethan, andererseits vor Substituirung anderer Urethane zu schützen. Nach Versuchen, die in der Merck'schen Fabrik, welche Urethan in grösseren Mengen für den medicinischen Bedarf darstellt, unternommen worden sind, schmilzt nämlich Methylurethan bei $52^{\circ}$, Propylurethan bei $53^{\circ}$ und Isoamylurethan bei $60^{\circ}$. Dabei erscheint es allerdings auffallend, dass hier eine Ausnahme von der Regel stattfinden soll, nach welcher sonst innerhalb homologer Reihen eine sich mit dem Ansteigen in der Reihe in gleicher Richtung bewegende Erhohung nicht nur der Siede-, sondern auch der Schmelzpunkte beobachtet wird. Ein erheblich unter $50^{\circ}$ liegender Schmelzpunkt ist bei einem zu relativ niederem Preise angebotenen Urethan anderer Provenienz constatirt worden. Dasselbe schmolz schon bei $44^{\circ}$ und erwies sich als stark wasserhaltig.

Wenden wir uns nun zu den physiologischen Eigenschaften, welche dem Urethan bei den Pharmakologen zu einer guten Meinung und zu einer so günstigen Prognose verholfen haben, dass manche glauben, dasselbe werde den Paraldehyd verdrängen. Vor letzterem hat es in erster Linie den nicht unangenehmen Geschmack und sehr leichte Löslichkait in Wasser voraus, so dass man das Mittel einfach in wässeriger Lösung ohne jedes Corrigens geben kann. Der Pharmakologe Schmiedeberg in Strassburg hat bezüg. lich der Wirkung an Thieren, Jolly an der dortigen psychiatrischen Klinik an Menschen Versuche angestellt, und beide, sowie auch v. Jackseh an der medicinischen Klinik in Wien constatirten, dass man es in dem Urethan mit einem vorzüglichen Hypnoticum zu thun habe, dessen Einführung in den Arzneischatz durchaus berechtigt sei. Der letztgenannte Kliniker hatte zunächst durch Thierversuche festgestellt, dass $0,5 \mathrm{~g}$. Urethan auf 1 Kilo Körpergewicht noch keine toxischen Erscheinungen hervorrufen, und dann bei 20 Kranken in 110 Einzelversuchen mit dem Mittel experimentirt. Hierbei hatte er mit keiner jener fatalen Nebenwirkungen zu käm- 
pfen, welche die Anwendung der meisten anderen bekannten Hypnotica so sehr erschweren. Dass Morphium häufig Erbrechen, Schwindel, Kopfschmerz hervorruft und deshalb mitunter nicht extragen wird, ist eime oft gemachte Erfahrung. Bei Chlorhydrat hat der Arzt mit der Möglichkeit des Eintretens von Herzschwäche zu rechnen, Verabreichung von Bromsalzen kann neben oft beobachteter Akne zuweilen selbst Bromkachexie herbeiführen, und gegen Paraldehyd haben viele Individuen einen ausgesprochenen Widerwillen. Nichts von alledem konnte in der Regel beim Gebrauch von Urethan wahrgenommen werden. Dasselbe wurde angewendet bei Lungentuberkulose, chronischem Gelenkrheumatismus, Carcinomen der Leber and des Rectums, Aorta aneurysma, Furunkulose, Gehirntumor, Rückenmarkstabes, Klappeninsufficienzen, Caries der Wirbelsäule u. s. w. und selten versagte es seine schlafbringende Wirkung, sobald die richtige Dosis von nicht unter $1 \mathrm{~g}$ gereicht wurde. Geringere Mengen von 0,25 und $0,5 \mathrm{~g}$ sind unsicher und verlangen öftere Wiederholung, jene grössere Gabe aber führt einen Schlaf herbei, welcher mit dem physiologischen die grösste Aehnlichkeit zeigen soll. Dagegen darf nicht verschwiegen werden, dass die Beeinflussung des Gehirns durch das Urethan nicht begleitet ist von einer solchen der Erregbarkeit des peripheren sensiblen Apparates, so dass heftige neuralgische Schmerzen, Athemnoth und ähnliche Zufälle dadurch nicht gemildert werden, wie dies wohl beim Morphiumgebrauch zu geschehen pflegt. Dafür fehlen aber auch auf der andern Seite die bekannten üblen Nachwirkungen des letzteren! Uebrigens ist nach Schmiedeberg das Urethan sowenig ein unfehlbares Universalmittel zur Hervorrufung von Schlaf, wie irgend eine andere Substanz.

Möge diese Besprechung eines in nächster Zeit wohl häufig arzneiliche Verwendung findenden Körpers Veranlassung zu weiteren chemisehen Versuchen mit demselben geben, besonders auch in der Richtung des Aufsuchens neuer Identitätsreaktionen und Prüfungsmethoden, damit dann die Pharmakopöecommission des Deutschen Apothekervereins, falls dieselbe auch dieses Mittel in den Kreis ihrer Arbeiten und Publikationen ziehen sollte, ein recht reichliches thatsächliches Material dazu vorfinde. 